\title{
Uma observação sobre as definições das funções seno e cosseno
}

\author{
Daniela Maria Grande Vicente(D) André VicenteiD
}

\begin{abstract}
Resumo
Em textos voltados ao ensino médio usualmente definem-se as funções seno e cosseno e, de modo geral, as funções trigonométricas, de maneira geométrica. Por outro lado, em textos de Análise Matemática definem-se seno e cosseno como sendo funções as quais envolvem limites de certas séries de potências. O objetivo deste trabalho é provar que as duas definições são equivalentes. A ideia principal é fazer a prova de maneira rigorosa mas sem perder o apelo geométrico envolvendo o uso do ciclo trigonométrico. Também faremos uma construção com régua e compasso, de modo a localizar (com uma certa precisão) os elementos das sequências de somas parciais das séries que definem cosseno (ou seno) no eixo dos cossenos (ou eixo dos senos).
\end{abstract}

Palavras-chave: Trigonometria; definição das funções seno e cosseno; Análise Matemática.

\begin{abstract}
In texts aimed at high school, the sine and cosine functions are usually defined, and in general the trigonometric functions, in a geometric way. On the other hand, in Mathematical Analysis texts, sine and cosine are defined as functions which involve limits of certain series of powers. The purpose of this work is to prove that the two definitions are equivalent. The main idea is to do the proof rigorously but without losing the geometric appeal involving the use of the trigonometric cycle. We will also make a construction with a ruler and compass in order to locate (with a certain precision) the elements of the sequence of partial sum of the series that define cosine (or sine) on the cosine axis (or sine axis).
\end{abstract}

Keywords: Trigonometric; definition of the functions sine and cosine; Mathematical Analysis.

\section{Introdução}

Em textos mais elementares, usualmente definem-se as funções trigonométricas de maneira geométrica. Precisamente, seja uv um sistema cartesiano ortogonal. Consideremos uma circunferência de raio 1 e centro na origem. Dado um número real $\mathrm{x}$, considere $\mathrm{P}=(\mathrm{a}, \mathrm{b})$ o ponto da circunferência tal que o arco compreendido entre $\mathrm{A}=(1,0)$ e $\mathrm{P}$ (medido no sentido anti-horário) tem medida $\mathrm{x}$. Define-se

$$
\cos \mathrm{x}=\mathrm{a} \quad \mathrm{e} \quad \operatorname{sen} \mathrm{x}=\mathrm{b}
$$


Isso permite-nos definir duas funções

$$
\cos : \mathbb{R} \rightarrow \mathbb{R} \quad \text { e } \quad \text { sen }: \mathbb{R} \rightarrow \mathbb{R}
$$

que associam, respectivamente, o número real $\mathrm{x}$ aos números reais $\mathrm{a}=\cos \mathrm{x}$ e $\mathrm{b}=\operatorname{sen} \mathrm{x}$. Ver Figura 1.

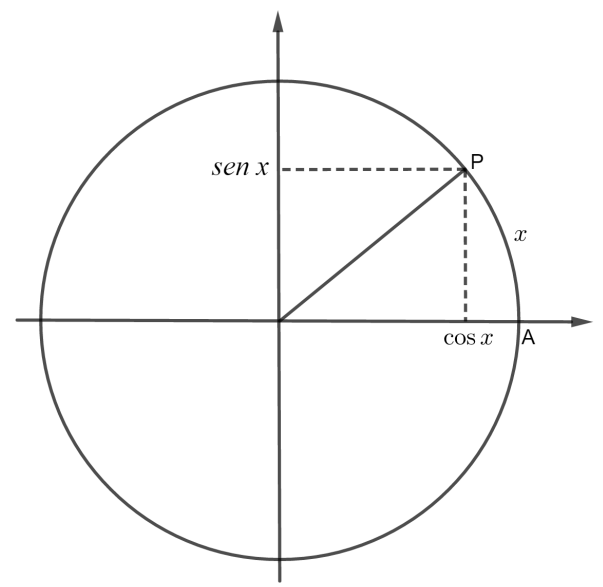

Figura 1: Tem-se que ( $\cos \mathrm{x}, \operatorname{sen} \mathrm{x})$ são as coordenadas do ponto $\mathrm{P}$ que pertence ao círculo de raio um e centro na origem do sistema coordenado.

Por outro lado, em textos de Análise Matemática definem-se cos e sen como sendo, respectivamente, as funções c $: \mathbb{R} \rightarrow \mathbb{R}$ e s $: \mathbb{R} \rightarrow \mathbb{R}$ dadas por

$$
c(x)=\sum_{n=0}^{\infty} \frac{(-1)^{n}}{(2 n) !} x^{2 n} \quad \text { e } \quad s(x)=\sum_{n=0}^{\infty} \frac{(-1)^{n}}{(2 n+1) !} x^{2 n+1} .
$$

O objetivo deste trabalho é provar que as duas definições acima são equivalentes, ou seja, provaremos que, para cada $x \in \mathbb{R}$, as sequências de somas parciais das séries definidas em (2) convergem para $\cos \mathrm{x}$ e sen $\mathrm{x}$, respectivamente, onde $\cos \mathrm{x}$ e sen $\mathrm{x}$ foram definidas em (1).

Uma prova desse fato pode ser encontrada em Figueiredo [1] (ver Teorema 4.5, página 109), onde o autor provou que existe um único par de funções $(f, g)$ definidas para todo $x \in \mathbb{R}$ tais que

$$
f^{\prime}(x)=g(x) \quad \text { e } \quad g^{\prime}(x)=-f(x)
$$

para todo $\mathrm{x} \in \mathbb{R}$, e

$$
\mathrm{f}(0)=0, \quad \text { e } \quad \mathrm{g}(0)=1 .
$$

Assim, como os pares (cos, sen ) e (c, s) satisfazem (3) e (4), conclui-se que as duas definições são equivalentes.

No entanto, como descrito acima, a prova apresentada em [1] traz-nos o resultado de maneira indireta e, por isso, não é muito intuitiva. A prova que apresentamos neste trabalho mostra tal resultado relacionando as sequências de somas parciais de $\mathrm{c}(\mathrm{x})$ e $\mathrm{s}(\mathrm{x}) \mathrm{com}$ o ciclo trigonométrico. 
Precisamente, dado um número real $\mathrm{x}$, se $\left(\mathrm{S}_{\mathrm{n}}(\mathrm{x})\right)_{\mathrm{n} \in \mathbb{N}}$ é a sequência de somas parciais de $\mathrm{c}(\mathrm{x})$ (com $\mathrm{c}(\mathrm{x})$ definida em (2)), localizamos os elementos do conjunto $\left\{\mathrm{S}_{0}(\mathrm{x}), \mathrm{S}_{1}(\mathrm{x}), \mathrm{S}_{2}(\mathrm{x}), \ldots, \mathrm{S}_{\mathrm{n}}(\mathrm{x}), \ldots\right\}$ no eixo dos cossenos para, posteriormente, provar que $\left(\mathrm{S}_{\mathrm{n}}(\mathrm{x})\right)_{\mathrm{n} \in \mathbb{N}}$ de fato converge para cos $\mathrm{x}$, ou seja, provamos que para cada $\mathrm{x} \in \mathbb{R}$

$$
c(x)=\lim _{n \rightarrow \infty} S_{n}(x)=\cos x,
$$

onde cos foi definido em (1). Além disso, faremos uma construção com régua e compasso para localizarmos, geometricamente, com uma certa precisão, os elementos de $\left\{\mathrm{S}_{0}(\mathrm{x}), \mathrm{S}_{1}(\mathrm{x}), \mathrm{S}_{2}(\mathrm{x}), \ldots\right.$, $\left.\mathrm{S}_{\mathrm{n}}(\mathrm{x}), \ldots\right\}$ no eixo dos cossenos. Provaremos que

$$
\mathrm{S}_{1}(\mathrm{x}) \leq \mathrm{S}_{3}(\mathrm{x}) \leq \mathrm{S}_{5}(\mathrm{x}) \leq \ldots \leq \mathrm{S}_{2 \mathrm{n}+1}(\mathrm{x}) \leq \ldots \leq \mathrm{S}_{2 \mathrm{n}}(\mathrm{x}) \leq \ldots \leq \mathrm{S}_{4}(\mathrm{x}) \leq \mathrm{S}_{2}(\mathrm{x}) \leq \mathrm{S}_{0}(\mathrm{x})
$$

e localizaremos os elementos no eixo dos cossenos conforme a Figura 2.

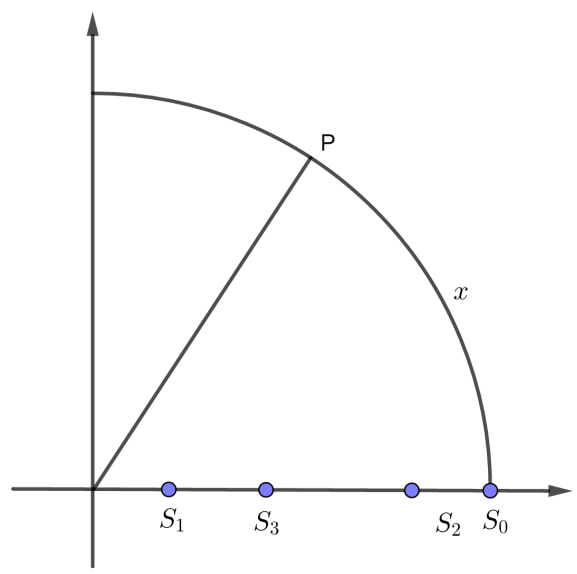

Figura 2: Provaremos algebricamente que os termos da sequência $\left(\mathrm{S}_{\mathrm{n}}(\mathrm{x})\right)_{\mathrm{n} \in \mathbb{N}}$ satisfazem $\mathrm{S}_{1}(\mathrm{x}) \leq$ $\mathrm{S}_{3}(\mathrm{x}) \leq \ldots \leq \mathrm{S}_{2 \mathrm{n}+1}(\mathrm{x}) \ldots \leq \mathrm{S}_{2 \mathrm{n}}(\mathrm{x}) \leq \ldots \leq \mathrm{S}_{2}(\mathrm{x}) \leq \mathrm{S}_{0}(\mathrm{x})$. Além disso, localizaremos os elementos da sequência no eixo dos cossenos.

O trabalho está organizado da seguinte forma: na Seção 2 apresentamos alguns resultados preliminares envolvendo convergência de sequências e séries; na Seção 3 provamos o resultado principal; e a Seção 4 é destinada à construção com régua e compasso dos termos da sequência de somas parciais $\left(\mathrm{S}_{\mathrm{n}}(\mathrm{x})\right)_{\mathrm{n} \in \mathbb{N}}$.

\section{Resultados preliminares}

Começamos esta seção lembrando o importante resultado envolvendo convergência de sequências numéricas o qual descreve que se $\left(a_{n}\right)_{n \in \mathbb{N}}$ é uma sequência monótona e limitada, então $\left(a_{n}\right)_{n \in \mathbb{N}}$ é convergente. A prova desse resultado pode ser encontrada, por exemplo, em Lima [5] e intrinsicamente envolve o fato de o conjunto dos números reais ser um corpo ordenado completo, característica que o distingue do conjunto dos números racionais. Se $\left(a_{n}\right)_{n \in \mathbb{N}}$ é uma sequência monótona crescente, então é possível provar que o limite da sequência é o número real

$$
\sup \left\{\mathrm{a}_{1}, \mathrm{a}_{2}, \mathrm{a}_{3}, \ldots, \mathrm{a}_{\mathrm{n}}, \ldots\right\}
$$


onde sup A denota o supremo do conjunto A. Analogamente, se $\left(\mathrm{a}_{\mathrm{n}}\right)_{\mathrm{n} \in \mathbb{N}}$ é monótona decrescente, temos que o limite é o número real

$$
\inf \left\{a_{1}, a_{2}, a_{3}, \ldots, a_{n}, \ldots\right\}
$$

onde inf A denota o ínfimo do conjunto A.

Dizemos que a série de potências $\sum_{\mathrm{k}=0}^{\infty} \mathrm{a}_{\mathrm{k}} \mathrm{x}^{\mathrm{k}}$ converge se a sequência de somas parciais $\left(\mathrm{R}_{\mathrm{n}}(\mathrm{x})\right)_{\mathrm{n} \in \mathbb{N}}$, onde $\mathrm{R}_{\mathrm{n}}(\mathrm{x})=\sum_{\mathrm{k}=0}^{\mathrm{n}} \mathrm{a}_{\mathrm{k}} \mathrm{x}^{\mathrm{k}}$, converge.

Aplicando o teste da razão (ver Lima [5], página 41 ou Muniz Neto [6], página 334) é possível provar que, para cada $\mathrm{x} \in \mathbb{R}$, as séries

$$
c(x)=\sum_{n=0}^{\infty} \frac{(-1)^{n}}{(2 n) !} x^{2 n} \quad \text { e } s(x)=\sum_{n=0}^{\infty} \frac{(-1)^{n}}{(2 n+1) !} x^{2 n+1}
$$

convergem. No entanto, aplicando o teste da razão diretamente perde-se o controle do valor do limite das séries, isto é, obtemos somente que a série converge sem saber qual é o valor do limite. Neste trabalho, para não perder tal controle, trabalhamos diretamente com as sequências de somas parciais das séries envolvidas. Assim, como as séries acima são alternadas, isto é, mudam de sinal a cada termo, uma maneira interessante de analisá-las é considerar as sequências de somas parciais de ordem par $\left(\mathrm{S}_{2 \mathrm{n}}(\mathrm{x})\right)_{\mathrm{n} \in \mathbb{N}}$ e de ordem ímpar $\left(\mathrm{S}_{2 \mathrm{n}+1}(\mathrm{x})\right)_{\mathrm{n} \in \mathbb{N}}$ separadamente.

É possível provar que, se $\left(R_{n}\right)_{n \in \mathbb{N}}$ é uma sequência tal que $\left(R_{2 n}\right)_{n \in \mathbb{N}}$ converge para $R$ e $\left(R_{2 n+1}\right)_{n \in \mathbb{N}}$ converge para $R$, então $\left(R_{n}\right)_{n \in \mathbb{N}}$ também converge para $R$. Usaremos esse fato na prova do resultado principal.

Por último, gostaríamos de lembrar o teorema de Rolle o qual garante que se $\mathrm{f}:[\mathrm{a}, \mathrm{b}] \rightarrow \mathbb{R}$ é uma função contínua em $[\mathrm{a}, \mathrm{b}]$ e derivável em $(\mathrm{a}, \mathrm{b})$ tal que $\mathrm{f}(\mathrm{a})=\mathrm{f}(\mathrm{b})$, então existe $\eta \in(\mathrm{a}, \mathrm{b})$ tal que $\mathrm{f}^{\prime}(\eta)=0$. Esse resultado é bastante intuitivo e uma interpretação geométrica é apresentada na Figura 3.

\section{Resultado principal}

Provaremos que

$$
c(x)=\sum_{n=0}^{\infty} \frac{(-1)^{n}}{(2 n) !} x^{2 n}=\lim _{n \rightarrow \infty} S_{n}(x)=\cos x .
$$

A prova de $\mathrm{s}(\mathrm{x})=$ sen $\mathrm{x}$ é análoga. Além disso, faremos o caso $\mathrm{x} \in\left(0, \frac{\pi}{2}\right)$. Com as devidas adaptações, as provas dos demais casos são análogas. A prova segue os seguintes passos:

1. Convergência de $\left(\mathrm{S}_{2 \mathrm{n}}(\mathrm{x})\right)_{\mathrm{n} \in \mathbb{N}}$ : Provaremos que $\left(\mathrm{S}_{2 \mathrm{n}}(\mathrm{x})\right)_{\mathrm{n} \in \mathbb{N}}$ é monótona decrescente e limitada, portanto é convergente, digamos, para $\mathrm{L}$ ( $\mathrm{L}$ depende de $\mathrm{x}$ ).

2. Relação entre $\left(\mathrm{S}_{2 \mathrm{n}}(\mathrm{x})\right)_{\mathrm{n} \in \mathbb{N}} e\left(\mathrm{~S}_{2 \mathrm{n}+1}(\mathrm{x})\right)_{\mathrm{n} \in \mathbb{N}}$ : Provaremos que

$$
\mathrm{S}_{2 \mathrm{n}}(\mathrm{x})>\mathrm{S}_{2 \mathrm{n}+1}(\mathrm{x})
$$

para todo $\mathrm{n} \in \mathbb{N}$ e para todo $\mathrm{x} \in\left(0, \frac{\pi}{2}\right)$. 


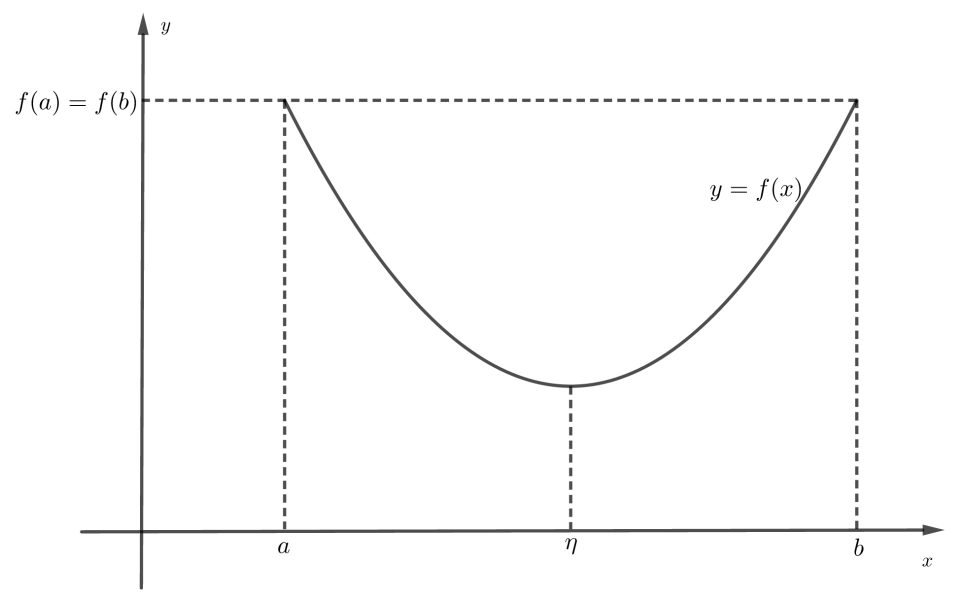

Figura 3: O teorema de Rolle garante que se $\mathrm{f}:[\mathrm{a}, \mathrm{b}] \rightarrow \mathbb{R}$ é uma função contínua em [a,b] e derivável em $(\mathrm{a}, \mathrm{b})$ tal que $\mathrm{f}(\mathrm{a})=\mathrm{f}(\mathrm{b})$, então existe $\eta \in(\mathrm{a}, \mathrm{b})$ tal que $\mathrm{f}^{\prime}(\eta)=0$.

3. Convergência de $\left(\mathrm{S}_{2 \mathrm{n}+1}(\mathrm{x})\right)_{\mathrm{n} \in \mathbb{N}}$ : Provaremos que $\left(\mathrm{S}_{2 \mathrm{n}+1}(\mathrm{x})\right)_{\mathrm{n} \in \mathbb{N}}$ é monótona crescente e limitada, portanto é convergente, digamos, para $\mathrm{M}$ ( $\mathrm{M}$ depende de $\mathrm{x})$.

4. Relação entre L, M e cosx: Provaremos que

$$
\cos \mathrm{x} \leq \mathrm{L} \leq \mathrm{S}_{2 \mathrm{n}}(\mathrm{x}) \leq 1 \quad \text { e } \quad \mathrm{S}_{2 \mathrm{n}+1}(\mathrm{x}) \leq \mathrm{M} \leq \cos \mathrm{x},
$$

para todo $\mathrm{n} \in \mathbb{N}$ e para todo $\mathrm{x} \in\left(0, \frac{\pi}{2}\right)$.

5. Conclusão da prova: Definiremos $\mathrm{d}=|\mathrm{M}-\mathrm{L}|$ e provaremos que

$$
\mathrm{d}=0 \text {. }
$$

Portanto, (8) e (9) permite-nos concluir que

$$
\lim _{n \rightarrow \infty} S_{2 n}(x)=L=\cos x=M=\lim _{n \rightarrow \infty} S_{2 n+1}(x),
$$

ou seja, as subsequências de somas parcias de ordem par e de ordem ímpar de $\left(\mathrm{S}_{\mathrm{n}}(\mathrm{x})\right)_{\mathrm{n} \in \mathbb{N}}$ convergem para L, consequentemente,

$$
\lim _{n \rightarrow \infty} S_{n}(x)=\cos x
$$

Inicialmente, observemos que o n-ésimo termo da sequência de somas parciais $\left(\mathrm{S}_{\mathrm{n}}(\mathrm{x})\right)_{\mathrm{n} \in \mathbb{N}}$ de $\sum_{n=0}^{\infty} \frac{(-1)^{n}}{(2 n) !} x^{2 n}$ é

$$
S_{n}(x)=1-\frac{x^{2}}{2 !}+\frac{x^{4}}{4 !}-\frac{x^{6}}{6 !}+\frac{x^{8}}{8 !}-\ldots+\frac{(-1)^{n}}{(2 n) !} x^{2 n} .
$$

Assim, os n-ésimos termos das sequências de somas parciais de ordem par e de ordem ímpar são, respectivamente,

$$
S_{2 n}(x)=1-\frac{x^{2}}{2 !}+\frac{x^{4}}{4 !}-\frac{x^{6}}{6 !}+\frac{x^{8}}{8 !}+\ldots-\frac{x^{4 n-2}}{(4 n-2) !}+\frac{x^{4 n}}{(4 n) !}
$$


e

$$
S_{2 n+1}(x)=1-\frac{x^{2}}{2 !}+\frac{x^{4}}{4 !}-\frac{x^{6}}{6 !}+\frac{x^{8}}{8 !}-\ldots+\frac{x^{4 n}}{(4 n) !}-\frac{x^{4 n+2}}{(4 n+2) !}
$$

Observe que

$$
S_{2 n+1}(x)=S_{2 n}(x)-\frac{x^{4 n+2}}{(4 n+2) !}
$$

para todo $\mathrm{n} \in \mathbb{N}$ e para todo $\mathrm{x} \in \mathbb{R}$.

Convergência de $\left(\mathrm{S}_{2 \mathrm{n}}(\mathrm{x})\right)_{\mathrm{n} \in \mathbb{N}}$ : Agora, provaremos que, para cada $\mathrm{x} \in\left(0, \frac{\pi}{2}\right)$, a sequência $\left(\mathrm{S}_{2 \mathrm{n}}(\mathrm{x})\right)_{\mathbb{N}}$ é monótona decrescente e limitada (portanto é convergente). Para isso, note que

$$
\begin{gathered}
S_{0}(x)=1, \\
S_{2}(x)=1-\frac{x^{2}}{2 !}+\frac{x^{4}}{4 !}=1-\frac{x^{2}}{2 !}\left(1-\frac{x^{2}}{4 \cdot 3}\right), \\
S_{4}(x)=1-\frac{x^{2}}{2 !}+\frac{x^{4}}{4 !}-\frac{x^{6}}{6 !}+\frac{x^{8}}{8 !}=1-\frac{x^{2}}{2 !}\left(1-\frac{x^{2}}{4 \cdot 3}\right)-\frac{x^{6}}{6 !}\left(1-\frac{x^{2}}{8 \cdot 7}\right), \\
S_{6}(x)=1-\frac{x^{2}}{2 !}+\frac{x^{4}}{4 !}-\frac{x^{6}}{6 !}+\frac{x^{8}}{8 !}-\frac{x^{10}}{10 !}+\frac{x^{12}}{12 !} \\
=1-\frac{x^{2}}{2 !}\left(1-\frac{x^{2}}{4 \cdot 3}\right)-\frac{x^{6}}{6 !}\left(1-\frac{x^{2}}{8 \cdot 7}\right)-\frac{x^{10}}{10 !}\left(1-\frac{x^{2}}{12 \cdot 11}\right) .
\end{gathered}
$$

Note também que

$$
4 \cdot 3>\frac{\pi}{2} \cdot \frac{\pi}{2}>x^{2}, \quad 8 \cdot 7>\frac{\pi}{2} \cdot \frac{\pi}{2}>x^{2} \text { e } 12 \cdot 11>\frac{\pi}{2} \cdot \frac{\pi}{2}>x^{2},
$$

para todo $\mathrm{x} \in\left(0, \frac{\pi}{2}\right)$, então

$$
1>\frac{x^{2}}{4 \cdot 3}, \quad 1>\frac{x^{2}}{8 \cdot 7} \text { e } 1>\frac{x^{2}}{12 \cdot 11},
$$

para todo $\mathrm{x} \in\left(0, \frac{\pi}{2}\right)$, consequentemente,

$$
\frac{x^{2}}{2 !}\left(1-\frac{x^{2}}{4 \cdot 3}\right)>0, \quad \frac{x^{6}}{6 !}\left(1-\frac{x^{2}}{8 \cdot 7}\right)>0 \quad \text { e } \frac{x^{10}}{10 !}\left(1-\frac{x^{2}}{12 \cdot 11}\right)>0
$$

para todo $\mathrm{x} \in\left(0, \frac{\pi}{2}\right)$. Portanto,

$$
\begin{aligned}
1=\mathrm{S}_{0}(\mathrm{x})> & \underbrace{\mathrm{S}_{0}(\mathrm{x})-\frac{\mathrm{x}^{2}}{2 !}\left(1-\frac{\mathrm{x}^{2}}{4 \cdot 3}\right)}_{=\mathrm{S}_{2}(\mathrm{x})}>\underbrace{\mathrm{S}_{2}(\mathrm{x})-\frac{\mathrm{x}^{6}}{6 !}\left(1-\frac{\mathrm{x}^{2}}{8 \cdot 7}\right)}_{=\mathrm{S}_{4}(\mathrm{x})} \\
& >\mathrm{S}_{4}(\mathrm{x})-\frac{\mathrm{x}^{10}}{10 !}\left(1-\frac{\mathrm{x}^{2}}{10 \cdot 9}\right)=\mathrm{S}_{6}(\mathrm{x}),
\end{aligned}
$$

para todo $\mathrm{x} \in\left(0, \frac{\pi}{2}\right)$, ou seja,

$$
\mathrm{S}_{0}(\mathrm{x})>\mathrm{S}_{2}(\mathrm{x})>\mathrm{S}_{4}(\mathrm{x})>\mathrm{S}_{6}(\mathrm{x}),
$$


para todo $\mathrm{x} \in\left(0, \frac{\pi}{2}\right)$. De modo geral os termos de $\left(\mathrm{S}_{2 \mathrm{n}}(\mathrm{x})\right)_{\mathrm{n} \in \mathbb{N}}$ podem ser escritos da seguinte forma:

$$
S_{2 n}(x)=1-\frac{x^{2}}{2 !}\left(1-\frac{x^{2}}{4 \cdot 3}\right)-\frac{x^{6}}{6 !}\left(1-\frac{x^{2}}{8 \cdot 7}\right)+\ldots-\frac{x^{4 n-2}}{(4 n-2) !}\left(1-\frac{x^{2}}{4 n \cdot(4 n-1)}\right)
$$

Para provar que a sequência é decrescente, basta mostrar que a contribuição que ocorre quando passamos do termo $\mathrm{S}_{2 \mathrm{n}}(\mathrm{x})$ para o termo $\mathrm{S}_{2 \mathrm{n}+2}(\mathrm{x})$ é negativa. Observando (12) e o argumento usado para concluir (11), devemos provar que ocorre

$$
1-\frac{x^{2}}{4 n \cdot(4 n-1)}>0
$$

para todo $\mathrm{n} \in \mathbb{N}$ e para todo $\mathrm{x} \in\left(0, \frac{\pi}{2}\right)$, mas isso é equivalente a

$$
16 n^{2}-4 n-x^{2}>0
$$

para todo $\mathrm{n} \in \mathbb{N}$ e para todo $\mathrm{x} \in\left(0, \frac{\pi}{2}\right)$, a qual é uma inequação do segundo grau. Temos que as raízes da equação $16 z^{2}-4 z-x^{2}=0$, na variável real z, são

$$
\mathrm{z}_{1}=\frac{1}{8}-\frac{\sqrt{1+4 \mathrm{x}^{2}}}{8} \text { e } \mathrm{z}_{2}=\frac{1}{8}+\frac{\sqrt{1+4 \mathrm{x}^{2}}}{8}
$$

Como $\mathrm{x} \in\left(0, \frac{\pi}{2}\right)$, temos que

$$
\frac{1}{8}<\frac{\sqrt{1+4 x^{2}}}{8}<\frac{\sqrt{1+4\left(\frac{\pi}{2}\right)^{2}}}{8}=\frac{\sqrt{1+\pi^{2}}}{8}
$$

Daqui,

$$
\mathrm{z}_{2}=\frac{1}{8}+\frac{\sqrt{1+4 \mathrm{x}^{2}}}{8}<\frac{1+\sqrt{1+\pi^{2}}}{8}<\frac{1+\sqrt{17}}{8}<\frac{1+\sqrt{25}}{8}=\frac{3}{4}<1 .
$$

Como $z_{1}<z_{2}$ e o gráfico de $y=16 z^{2}-4 z-x^{2}$ é uma parábola com a concavidade voltada para cima, concluímos que $16 z^{2}-4 z-x^{2}$ é negativo somente quando z está entre $z_{1}$ e $z_{2}$. Como $z_{2}<1$, concluímos que a desigualdade (14) ocorre para todo $\mathrm{n} \in \mathbb{N}$, como queríamos; ver Figura 4 . Isso prova que (13) ocorre, ou seja, que o termo que é adicionado quando passamos de $\left(\mathrm{S}_{2 \mathrm{n}}(\mathrm{x})\right)_{\mathrm{n} \in \mathbb{N}}$ para $\left(\mathrm{S}_{2 \mathrm{n}+2}(\mathrm{x})\right)_{\mathrm{n} \in \mathbb{N}}$ é negativo. Assim, $\left(\mathrm{S}_{2 \mathrm{n}}(\mathrm{x})\right)_{\mathrm{n} \in \mathbb{N}}$ é decrescente. Temos também que a sequência é limitada, pois

$$
0<\ldots<\mathrm{S}_{2 \mathrm{n}}(\mathrm{x})<\mathrm{S}_{2 \mathrm{n}-2}(\mathrm{x})<\ldots<\mathrm{S}_{2}(\mathrm{x})<\mathrm{S}_{0}(\mathrm{x})=1,
$$

para todo $\mathrm{x} \in\left(0, \frac{\pi}{2}\right)$. Assim, $\left(\mathrm{S}_{2 \mathrm{n}}(\mathrm{x})\right)_{\mathrm{n} \in \mathbb{N}}$ converge para

$$
\mathrm{L}:=\inf \left\{\mathrm{S}_{0}(\mathrm{x}), \mathrm{S}_{2}(\mathrm{x}), \mathrm{S}_{4}(\mathrm{x}), \mathrm{S}_{6}(\mathrm{x}), \ldots, \mathrm{S}_{2 \mathrm{n}}(\mathrm{x}), \ldots\right\}
$$

aqui L depende de x, mas, para simplificar a notação, omitiremos a dependência. Geometricamente, temos a situação da Figura 5.

Relação entre $\left(\mathrm{S}_{2 \mathrm{n}}(\mathrm{x})\right)_{\mathrm{n} \in \mathbb{N}}$ e $\left(\mathrm{S}_{2 \mathrm{n}+1}(\mathrm{x})\right)_{\mathrm{n} \in \mathbb{N}}$ : Note que

$$
\mathrm{S}_{2 \mathrm{n}}(\mathrm{x})>\mathrm{S}_{2 \mathrm{n}+1}(\mathrm{x})
$$




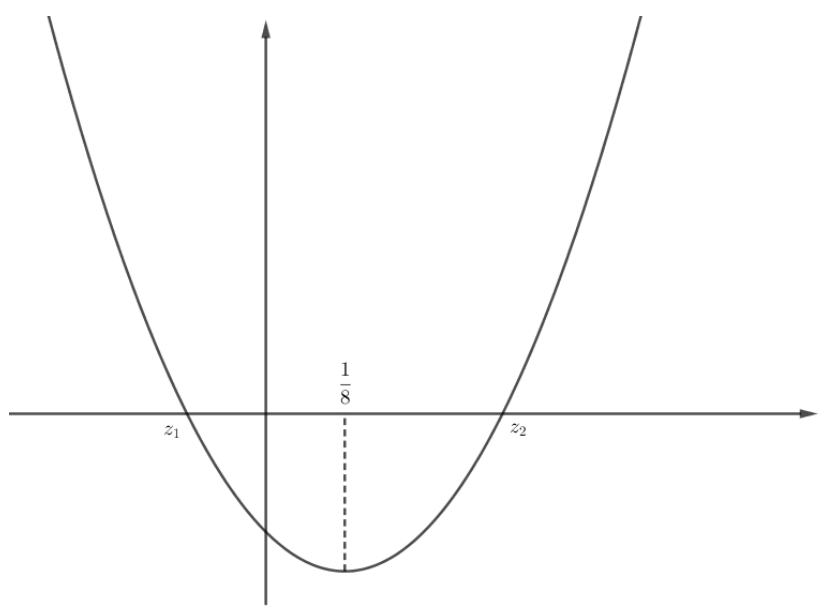

Figura 4: As raízes da equação $y=16 z^{2}-4 z-x^{2}$ são $z_{1}=\frac{1}{8}-\frac{\sqrt{1+4 x^{2}}}{8}$ e $z_{2}=\frac{1}{8}+\frac{\sqrt{1+4 x^{2}}}{8}$. Note que, como $\mathrm{x} \in\left(0, \frac{\pi}{2}\right), \mathrm{z}_{1}<0<\mathrm{z}_{2}<1$. Portanto, $16 \mathrm{n}^{2}-4 \mathrm{n}-\mathrm{x}^{2}>0$, para todo $\mathrm{n} \in \mathbb{N}$.

para todo $\mathrm{n} \in \mathbb{N}$, pois de (10)

$$
\mathrm{S}_{2 \mathrm{n}+1}(\mathrm{x})-\mathrm{S}_{2 \mathrm{n}}(\mathrm{x})=-\frac{\mathrm{x}^{4 \mathrm{n}+2}}{(4 \mathrm{n}+2) !}<0
$$

para todo $\mathrm{n} \in \mathbb{N}$ e para todo $\mathrm{x} \in\left(0, \frac{\pi}{2}\right)$.

Convergência de $\left(\mathrm{S}_{2 \mathrm{n}+1}(\mathrm{x})\right)_{\mathrm{n} \in \mathbb{N}}$ : Procedendo da mesma forma que no caso da sequência de somas parciais formada pelos termos de ordem par, prova-se que $\left(\mathrm{S}_{2 \mathrm{n}+1}(\mathrm{x})\right)_{\mathrm{n} \in \mathbb{N}}$ é monótona crescente e limitada. Para isso, basta agrupar os termos da sequência da seguinte forma

$$
S_{2 n+1}(x)=1-\frac{x^{2}}{2 !}+\frac{x^{4}}{4 !}\left(1-\frac{x^{2}}{6 \cdot 5}\right)+\frac{x^{8}}{8 !}\left(1-\frac{x^{2}}{10 \cdot 9}\right)+\frac{x^{4 n}}{(4 n) !}\left(1-\frac{x^{2}}{(4 n+2) \cdot(4 n+1)}\right)
$$

e usar os argumentos do caso $\left(\mathrm{S}_{2 \mathrm{n}}(\mathrm{x})\right)_{\mathrm{n} \in \mathbb{N}}$. Assim, $\left(\mathrm{S}_{2 \mathrm{n}+1}(\mathrm{x})\right)_{\mathrm{n} \in \mathbb{N}}$ converge para

$$
M:=\sup \left\{\mathrm{S}_{1}(\mathrm{x}), \mathrm{S}_{3}(\mathrm{x}), \mathrm{S}_{5}(\mathrm{x}), \ldots, \mathrm{S}_{2 \mathrm{n}+1}(\mathrm{x}), \ldots\right\},
$$

temos que $\mathrm{M}$ depende de $\mathrm{x}$, para simplicar a notação não deixaremos esta dependência explícita. Geometricamente, temos a situação da Figura 6.

Relação entre L, M e cosx: A próxima etapa é provar que

$$
\cos \mathrm{x} \leq \mathrm{L} \leq \mathrm{S}_{2 \mathrm{n}}(\mathrm{x}) \leq 1,
$$

para todo $\mathrm{n} \in \mathbb{N}$ e para todo $\mathrm{x} \in\left(0, \frac{\pi}{2}\right)$. Para isso, seja $\mathrm{x} \in\left(0, \frac{\pi}{2}\right)$ um número arbitrariamente fixado e defina $g:[0, x] \rightarrow \mathbb{R}$ por $\mathrm{g}(\lambda)=\cos \mathrm{x}-\cos \lambda+(\mathrm{x}-\lambda) \operatorname{sen} \lambda+(\mathrm{x}-\lambda)^{2} \frac{\cos \lambda}{2}-(\mathrm{x}-\lambda)^{3} \frac{\operatorname{sen} \lambda}{3 !}-(\mathrm{x}-\lambda)^{4} \frac{\cos \lambda}{4 !}+(\mathrm{x}-\lambda)^{5} \frac{\operatorname{sen} \lambda}{5 !}$ $+\ldots-(\mathrm{x}-\lambda)^{4 \mathrm{n}} \frac{\cos \lambda}{(4 \mathrm{n}) !}-(\mathrm{x}-\lambda)^{4 \mathrm{n}+1} \frac{\mathrm{K}}{(4 \mathrm{n}+1) !}$, 


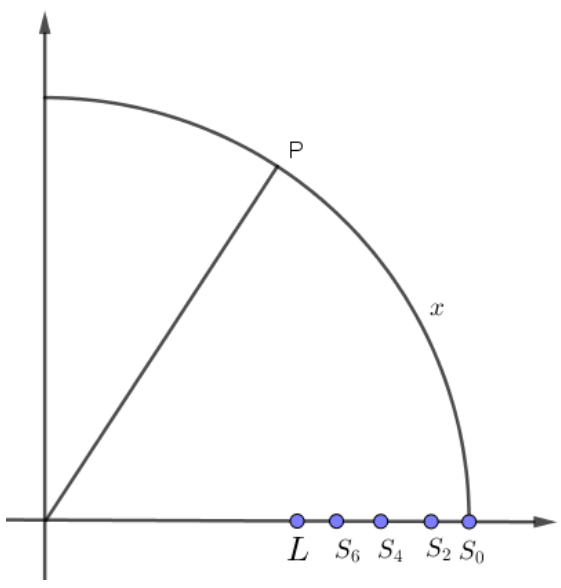

Figura 5: Os elementos da sequência de somas parciais de ordem par $\left(\mathrm{S}_{2 \mathrm{n}}(\mathrm{x})\right)_{\mathrm{n} \in \mathbb{N}}$ é uma sequência monótona decrescente.

onde $\mathrm{K}$ é uma constante fixa tal que

$$
g(0)=0 .
$$

Derivando g obtemos que

$$
\mathrm{g}^{\prime}(\lambda)=\frac{\mathrm{K}+\operatorname{sen} \lambda}{(4 \mathrm{n}) !}(\mathrm{x}-\lambda)^{4 \mathrm{n}}
$$

Por outro lado, como g é contínua em [0, $\mathrm{x}]$, derivável em $(0, \mathrm{x})$ e $\mathrm{g}(0)=\mathrm{g}(\mathrm{x})=0$, podemos aplicar o teorema de Rolle e concluir que existe uma constante $\eta_{0} \in(0, \mathrm{x})$ tal que

$$
\mathrm{g}^{\prime}\left(\eta_{0}\right)=0
$$

De (19) e (20) temos que

$$
\frac{\mathrm{K}+\operatorname{sen} \eta_{0}}{(4 \mathrm{n}) !}\left(\mathrm{x}-\eta_{0}\right)^{4 \mathrm{n}}=0 .
$$

Mas, como $\eta_{0}<\mathrm{x}$, essa igualdade é possível somente quando

$$
\mathrm{K}=-\operatorname{sen} \eta_{0}
$$

Observando a definição de g, (18) e (21), temos que

$$
0=g(0)=\cos x-1+\frac{x^{2}}{2 !}-\frac{x^{4}}{4 !}+\frac{x^{6}}{6 !}-\frac{x^{8}}{8 !}-\ldots-\frac{x^{4 n}}{(4 n) !}+\frac{\operatorname{sen} \eta_{0}}{(4 n+1) !} x^{4 n+1} .
$$

Disso, e observando a definição de $\mathrm{S}_{2 \mathrm{n}}(\mathrm{x})$, chegamos a

$$
\cos \mathrm{x}-\mathrm{S}_{2 \mathrm{n}}(\mathrm{x})+\frac{\operatorname{sen} \eta_{0}}{(4 \mathrm{n}+1) !} \mathrm{x}^{4 \mathrm{n}+1}=0
$$

Daqui, e como $\eta_{0} \in\left(0, \frac{\pi}{2}\right)$, temos que

$$
\cos \mathrm{x}-\mathrm{S}_{2 \mathrm{n}}(\mathrm{x})=-\frac{\operatorname{sen} \eta_{0}}{(4 \mathrm{n}+1) !} \mathrm{x}^{4 \mathrm{n}+1}<0 .
$$




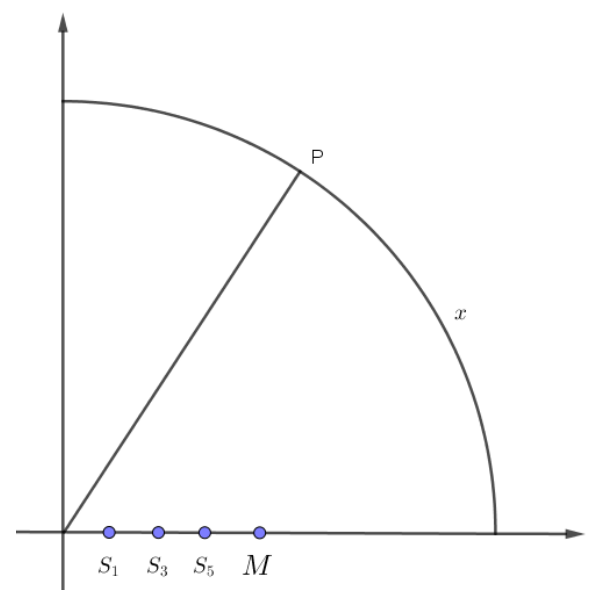

Figura 6: Os elementos da sequência de somas parciais de ordem ímpar $\left(\mathrm{S}_{2 \mathrm{n}+1}(\mathrm{x})\right)_{\mathrm{n} \in \mathbb{N}}$ são uma sequência monótona crescente.

Portanto,

$$
\cos \mathrm{x}<\mathrm{S}_{2 \mathrm{n}}(\mathrm{x}) .
$$

Como $\mathrm{n} \in \mathbb{N}$ e $\mathrm{x} \in\left(0, \frac{\pi}{2}\right)$ são arbitrários, temos que (22) ocorre para todo $\mathrm{n} \in \mathbb{N}$ e para todo $\mathrm{x} \in\left(0, \frac{\pi}{2}\right)$. A estimativa (22) fornece-nos que cos $\mathrm{x}$ é uma cota inferior de $\left\{\mathrm{S}_{0}(\mathrm{x}), \mathrm{S}_{2}(\mathrm{x}), \mathrm{S}_{4}(\mathrm{x})\right.$, $\left.\mathrm{S}_{6}(\mathrm{x}), \ldots, \mathrm{S}_{2 \mathrm{n}}(\mathrm{x}), \ldots\right\}$; assim,

$$
\cos \mathrm{x} \leq \mathrm{L},
$$

pois $\mathrm{L}=\inf \left\{\mathrm{S}_{0}(\mathrm{x}), \mathrm{S}_{2}(\mathrm{x}), \mathrm{S}_{4}(\mathrm{x}), \mathrm{S}_{6}(\mathrm{x}), \ldots, \mathrm{S}_{2 \mathrm{n}}(\mathrm{x}), \ldots\right\}$. Ainda, (23) e a definição de $\mathrm{L}$ permite-nos inferir que

$$
\cos \mathrm{x} \leq \mathrm{L} \leq \mathrm{S}_{2 \mathrm{n}}(\mathrm{x})
$$

para todo $\mathrm{x} \in\left(0, \frac{\pi}{2}\right)$. Além disso, não é difícil verificar que $\mathrm{S}_{2 \mathrm{n}}(\mathrm{x}) \leq 1$, para todo $\mathrm{x} \in\left(0, \frac{\pi}{2}\right)$. Portanto, (17) está provado.

Analogamente, prova-se que

$$
\mathrm{S}_{2 \mathrm{n}+1}(\mathrm{x}) \leq \mathrm{M} \leq \cos \mathrm{x}
$$

para todo $\mathrm{n} \in \mathbb{N}$ e para todo $\mathrm{x} \in\left(0, \frac{\pi}{2}\right)$.

Geometricamente, (24) e (25) fornecem-nos a situação representada na Figura 7.

Conclusão da prova: Provaremos agora que $\mathrm{L}=\mathrm{M}$, ou seja, que as sequências de somas parciais de ordem par e de ordem ímpar de $\left(\mathrm{S}_{\mathrm{n}}(\mathrm{x})\right)_{\mathrm{n} \in \mathbb{N}}$ convergem para o mesmo valor, consequentemente $\left(\mathrm{S}_{\mathrm{n}}(\mathrm{x})\right)_{\mathrm{n} \in \mathbb{N}}$ também converge para este valor. A prova também nos fornece o valor do limite, isto é, provamos que $\lim _{\mathrm{n} \rightarrow \infty} \mathrm{S}_{\mathrm{n}}(\mathrm{x})=\cos \mathrm{x}$, que é o resultado principal desse trabalho.

De fato, de (17) e (25) temos que

$$
\mathrm{S}_{2 \mathrm{n}+1}(\mathrm{x}) \leq \mathrm{M} \leq \cos \mathrm{x} \leq \mathrm{L} \leq \mathrm{S}_{2 \mathrm{n}}(\mathrm{x}) .
$$

Seja

$$
\mathrm{d}=|\mathrm{M}-\mathrm{L}|,
$$




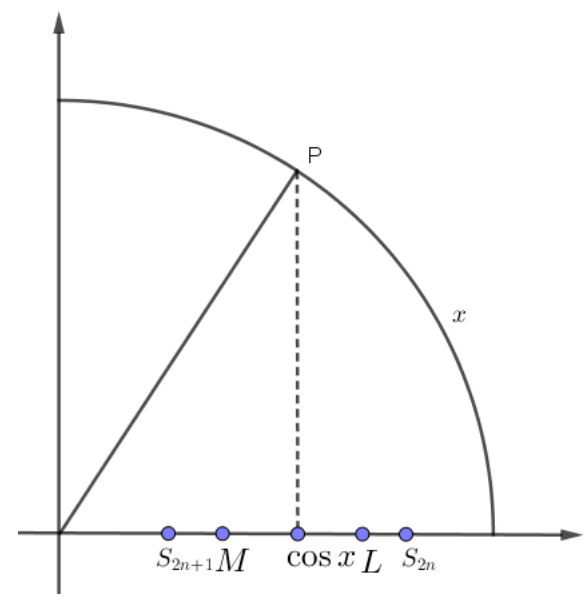

Figura 7: De (24) e (25) temos que $\mathrm{S}_{2 \mathrm{n}+1}(\mathrm{x}) \leq \mathrm{M} \leq \cos \mathrm{x} \leq \mathrm{L} \leq \mathrm{S}_{2 \mathrm{n}}(\mathrm{x})$.

onde $|z|$ é o valor absoluto do número real z. Ver Figura 8.

Assim, observando (10) e (26) temos que

$$
d=|M-L| \leq\left|S_{2 n+1}(x)-S_{2 n}(x)\right|=\frac{x^{4 n+2}}{(4 n+2) !}<\frac{\left(\frac{\pi}{2}\right)^{4 n+2}}{(4 n+2) !}<\frac{\left(\frac{4}{2}\right)^{4 n+2}}{(4 n+2) !}=\frac{2^{4 n+2}}{(4 n+2) !},
$$

para todo $\mathrm{x} \in\left(0, \frac{\pi}{2}\right)$ e para todo $\mathrm{n} \in \mathbb{N}$. Note que

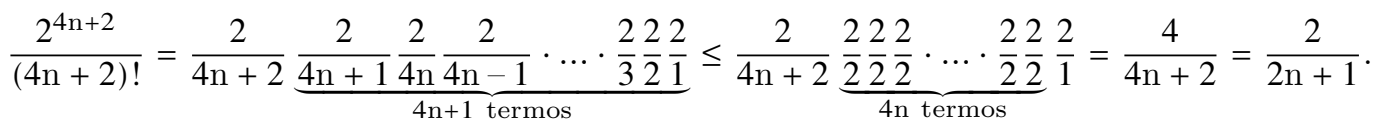

Logo,

$$
\mathrm{d}=|\mathrm{M}-\mathrm{L}|<\frac{2}{2 \mathrm{n}+1},
$$

para todo $n \in \mathbb{N}$. Como $d=|M-L|$, temos que $d \geq 0$. Se $d>0$, então escolhendo $n \in \mathbb{N}$ tal que

$$
\mathrm{n} \geq \frac{4-\mathrm{d}}{2 \mathrm{~d}}
$$

de onde concluímos que

$$
\frac{2}{2 n+1} \leq \frac{d}{2}
$$

Portanto, (27) e (28) fornecem-nos que

$$
\mathrm{d}=|\mathrm{M}-\mathrm{L}|<\frac{\mathrm{d}}{2}
$$

o que não é possível. Portanto, ocorre que $\mathrm{d}=0$, consequentemente $\mathrm{L}=\mathrm{M}$. Disso, e observando (26), concluímos também que $\mathrm{L}=\cos \mathrm{x}$, como queríamos provar. 


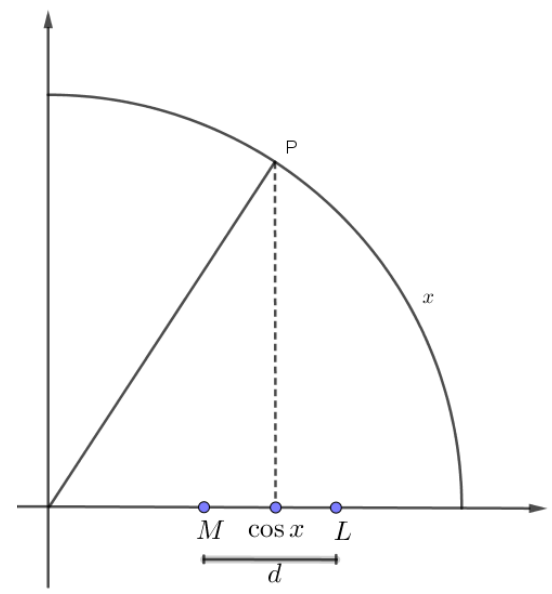

Figura 8: O número d é a distância entre L e M.

\section{Construção geométrica de $\mathrm{S}_{\mathrm{n}}(\mathrm{x})$}

O objetivo dessa seção é indicar como pode ser feita a construção dos elementos do conjunto

$$
\left\{\mathrm{S}_{0}(\mathrm{x}), \mathrm{S}_{1}(\mathrm{x}), \mathrm{S}_{2}(\mathrm{x}), \mathrm{S}_{3}(\mathrm{x}), \mathrm{S}_{4}(\mathrm{x}), \ldots\right\}
$$

com régua e compasso. Além disso, localizaremos $\mathrm{S}_{0}(\mathrm{x}), \mathrm{S}_{1}(\mathrm{x})$ e $\mathrm{S}_{2}(\mathrm{x})$ no eixo dos cossenos. Para detalhes sobre construções geométricas com regra e compasso, ver Putnoki [3, 4] e Silva Junior [2]. Assim, seja $\operatorname{arc}(\mathrm{AP})$ um arco, compreendido entre $\mathrm{A}=(1,0)$ e $\mathrm{P}=(\cos \mathrm{x}, \operatorname{sen} \mathrm{x})$, de comprimento $\mathrm{x}, \operatorname{com} \mathrm{x} \in\left(0, \frac{\pi}{2}\right)$ qualquer. A construção apresentada abaixo está separada em vii) etapas. Cada etapa será uma continuação da anterior e será feita sem uma nova descrição dos elementos geométricos já construídos.

i) Construção de um segmento de comprimento $\mathrm{x}$ : A primeira etapa é retificar o $\operatorname{arco} \operatorname{arc}(\mathrm{AP})$. Para isso denotemos por $\mathrm{B}$ o ponto da circunferência trigonométrica de coordenadas $(-1,0)$ e $\mathrm{C}$ o ponto que pertence à reta $\mathrm{r}$ que passa por $\mathrm{A}$ e $\mathrm{B}$ tal que a distância entre $\mathrm{B}$ e $\mathrm{C}$ é $\frac{3}{4}$.

Construa uma reta s perpendicular à reta $\mathrm{r}$ e que passe por A. Construa uma reta que passe por $\mathrm{C}$ e P. Seja $\mathrm{P}^{\prime}$ o ponto de intersecção dessa reta com a reta s. Ver Figura 9.

Verifica-se que o comprimento do segmento $\mathrm{AP}^{\prime}$ é uma aproximação razoável do comprimento do $\operatorname{arco} \operatorname{arc}(\mathrm{AP})$. Precisamente, prova-se que

$$
\overline{\mathrm{AP}^{\prime}}=\frac{11 \operatorname{sen} \mathrm{x}}{7+4 \cos \mathrm{x}}
$$

onde $\overline{\mathrm{AP}^{\prime}}$ denota o comprimento do segmento $\mathrm{AP}^{\prime}$. De fato, seja $\mathrm{D}=(\cos \mathrm{x}, 0)$. Tem-se que os triângulos $\mathrm{CDP}$ e $\mathrm{CAP}^{\prime}$ são semelhantes. Assim,

$$
\frac{\overline{\mathrm{AP}^{\prime}}}{\overline{\mathrm{AC}}}=\frac{\overline{\mathrm{DP}}}{\overline{\mathrm{CD}}} .
$$




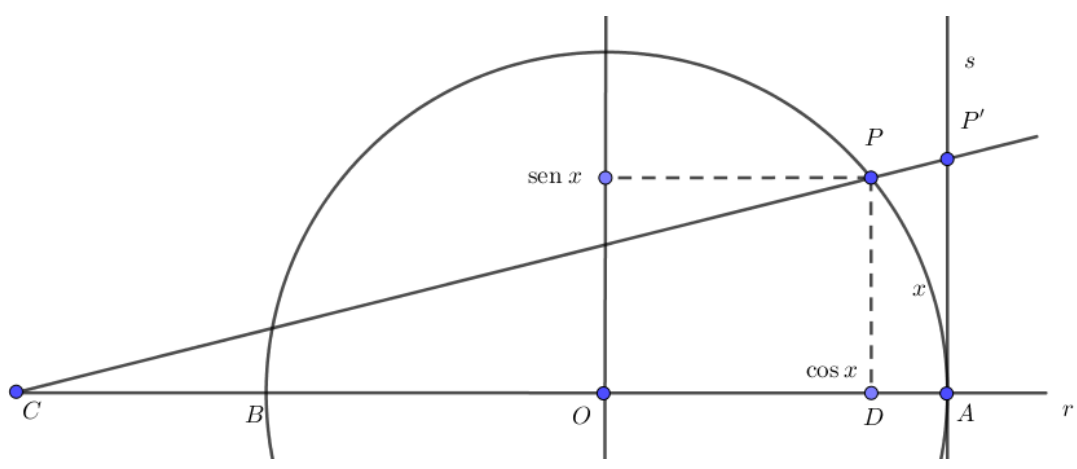

Figura 9: $\mathrm{O}$ arco $\operatorname{arc}(\mathrm{AP})$ tem medida $\mathrm{x}$, e o comprimento do segmento $\mathrm{AP}^{\prime}$ é uma aproximação do comprimento do $\operatorname{arco} \operatorname{arc}(\mathrm{AP})$.

Disso,

$$
\frac{\overline{\mathrm{AP}^{\prime}}}{\frac{11}{4}}=\frac{\text { sen } \mathrm{x}}{\overline{\mathrm{CO}}+\overline{\mathrm{OD}}},
$$

ou seja,

$$
\frac{\overline{\mathrm{AP}^{\prime}}}{\frac{11}{4}}=\frac{\operatorname{sen} \mathrm{x}}{\frac{7}{4}+\cos \mathrm{x}}
$$

disso, conclui-se que (29) ocorre.

Como sabemos que $\operatorname{arc}(\mathrm{AP})=\mathrm{x}$, então

$$
\lambda:=\left|\operatorname{arc}(\mathrm{AP})-\overline{\mathrm{AP}^{\prime}}\right|=\left|\mathrm{x}-\frac{11 \operatorname{sen} \mathrm{x}}{7+4 \cos \mathrm{x}}\right| .
$$

Por exemplo, para $\mathrm{x}=\frac{\pi}{6}, \frac{\pi}{4}$ e $\frac{\pi}{3}$ os valores aproximados de $\lambda$ são, respectivamente, 0,002008 , 0,006 e 0,01 .

ii) Construção de um segmento de comprimento $\mathrm{x}^{2}$ : Com a construção do segmento $\mathrm{AP}^{\prime}$ com comprimento (aproximado) de $\mathrm{x}$, o próximo passo é construir um segmento de comprimento $\mathrm{x}^{2}$. Para isso, seja $\mathrm{D}$ um ponto de $\mathrm{r}$ tal que $\overline{\mathrm{AD}}=\overline{\mathrm{AP}^{\prime}}$. Construa também o ponto $\mathrm{E}$ em $\mathrm{s}$ tal que $\overline{\mathrm{AE}}=1$. Seja t a reta que passa por $\mathrm{D}$ e $\mathrm{E}$ e $\mathrm{u}$ a reta paralela a $\mathrm{t}$ e que passa por $\mathrm{P}^{\prime}$. Denotemos por $\mathrm{F}$ o ponto de intersecção das retas r e u. Conforme Figura 10.

Note que os triângulos $\mathrm{ADE}$ e $\mathrm{AFP}^{\prime}$ são semelhantes. Assim,

$$
\frac{\overline{\mathrm{AD}}}{\overline{\mathrm{AE}}}=\frac{\overline{\mathrm{AF}}}{\overline{\overline{\mathrm{AP}^{\prime}}}} ;
$$

daqui,

$$
\frac{x}{1}=\frac{\overline{A F}}{x},
$$

ou seja,

$$
\overline{\mathrm{AF}}=\mathrm{x}^{2} .
$$




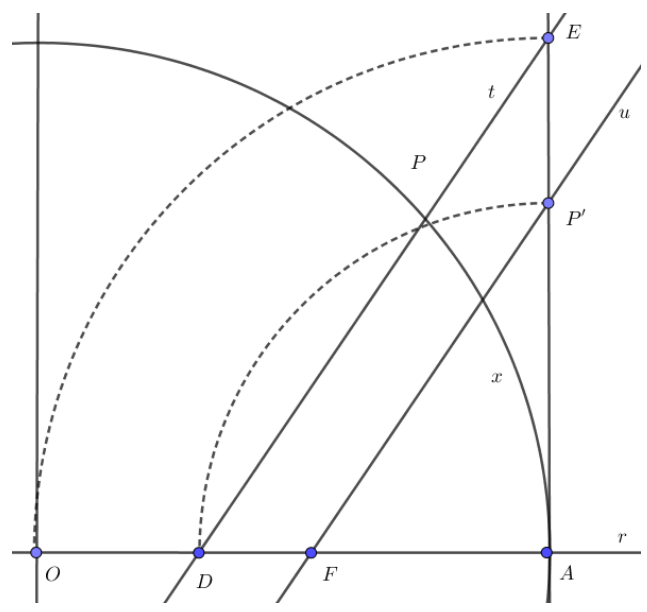

Figura 10: A medida do segmento AF é $\mathrm{x}^{2}$.

iii) Construção de um segmento de comprimento $\frac{\mathrm{x}^{2}}{2}$ : Traçando a mediatriz do segmento AF obtemos o ponto $\mathrm{G}$ tal que

$$
\overline{\mathrm{FG}}=\overline{\mathrm{AG}}=\frac{\mathrm{x}^{2}}{2} .
$$

Ver Figura 11.

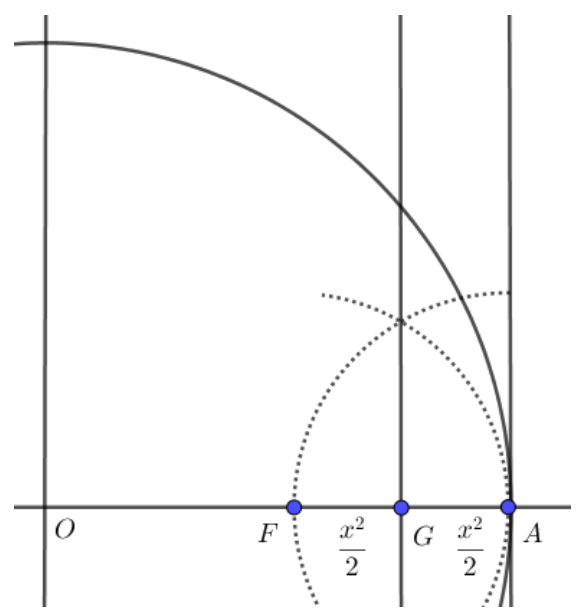

Figura 11: A medida dos segmentos FG e AG é $\frac{x^{2}}{2}$.

iv) Localização de $\mathrm{S}_{1}(\mathrm{x})$ : Observando a definição de $\mathrm{S}_{1}(\mathrm{x})$ e a construção acima concluímos que

$$
\overline{\mathrm{OG}}=1-\frac{\mathrm{x}^{2}}{2}=\mathrm{S}_{1}(\mathrm{x}) .
$$


Ver Figura 12.



Figura 12: A medida do segmento OG é $\mathrm{S}_{1}(\mathrm{x})=1-\frac{\mathrm{x}^{2}}{2}$.

v) Construção de um segmento de comprimento $\frac{\mathrm{x}^{4}}{4}$ : Para a construção de $\mathrm{S}_{2}(\mathrm{x})$ é necessário construir um segmento de comprimento $\frac{\mathrm{x}^{4}}{4}$. Assim, começamos traçando a reta $\mathrm{v}$ que passe por $\mathrm{G}$ e é perpendicular a $\mathrm{r}$. Marcamos em v o ponto $\mathrm{H}$ tal que $\overline{\mathrm{GH}}=\overline{\mathrm{AG}}=\frac{\mathrm{x}^{2}}{2}$. Seja I o ponto de $\mathrm{v}$ tal que $\overline{\mathrm{GI}}=\overline{\mathrm{OA}}=1$. Tracemos uma reta que passe por $\mathrm{H}$ e é paralela à reta que passa por I e A. Seja J o ponto de intersecção dessa reta com a reta r. Temos que os triângulos AGI e JGH são semelhantes. Ver Figura 13. Assim, temos que

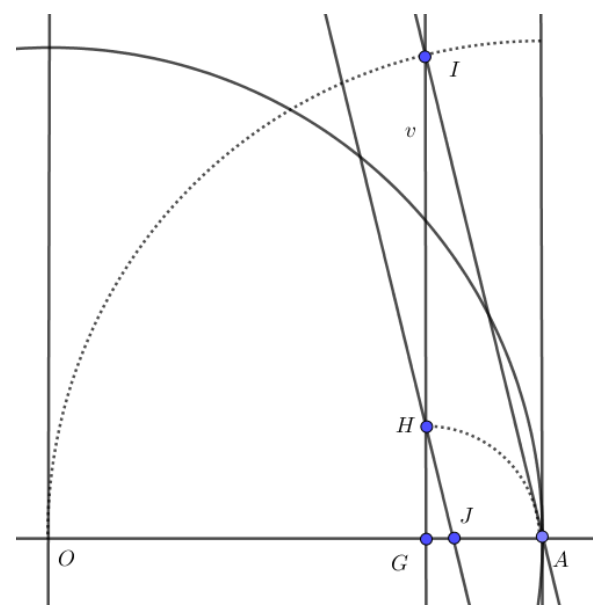

Figura 13: A medida do segmento GJ é $\frac{x^{4}}{4}$. 


$$
\frac{\overline{\mathrm{GI}}}{\overline{\mathrm{GH}}}=\frac{\overline{\mathrm{AG}}}{\overline{\mathrm{GJ}}} ;
$$

daqui,

$$
\frac{1}{\frac{x^{2}}{2}}=\frac{\frac{x^{2}}{2}}{\overline{G J}},
$$

ou seja,

$$
\overline{\mathrm{GJ}}=\frac{\mathrm{x}^{4}}{4}
$$

vi) Construção de um segmento de comprimento $\frac{\mathrm{x}^{4}}{4 !}$ : Seja $\mathrm{K}$ o ponto em v tal que $\overline{\mathrm{GK}}=6=3 \cdot 2$. Tracemos uma reta que passe por I e seja paralela à reta que passa por J e K. Seja L o ponto de intersecção dessa reta com a reta r. Então os triângulos GJK e GLI são semelhantes. Ver Figura 14. Assim, temos que

$$
\frac{\overline{\mathrm{GK}}}{\overline{\mathrm{GI}}}=\frac{\overline{\mathrm{GJ}}}{\overline{\overline{\mathrm{GL}}}},
$$

$\log \mathrm{O}$

$$
\frac{6}{1}=\frac{\frac{x^{4}}{4}}{\overline{G L}},
$$

portanto,

$$
\overline{\mathrm{GL}}=\frac{\mathrm{x}^{4}}{4 !} .
$$

Ver Figura 14. Note que a Figura 14 não mantém proporcinalidade na construção.

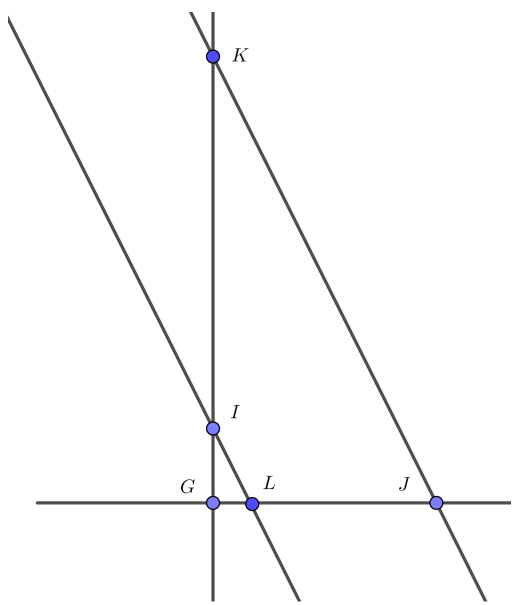

Figura 14: Os triângulos GJK e GLI são semelhantes. O ponto G é marcado de forma que a medida de GK é $6=3 \cdot 2$. A medida de GL é $\frac{x^{4}}{4 !}$. 
vii) Localização de $\mathrm{S}_{2}(\mathrm{x})$ : Da construção acima, concluímos que

$$
\overline{\mathrm{OL}}=\overline{\mathrm{OG}}+\overline{\mathrm{GL}}=\left(1-\frac{\mathrm{x}^{2}}{2}\right)+\frac{\mathrm{x}^{4}}{4 !}=\mathrm{S}_{2}(\mathrm{x}) .
$$

Ver Figura 15.

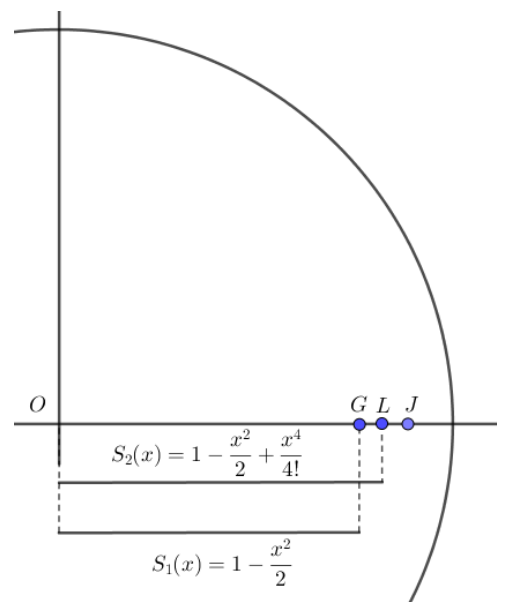

Figura 15: A medida do segmento OL é $\mathrm{S}_{2}(\mathrm{x})=1-\frac{\mathrm{x}^{2}}{2}+\frac{\mathrm{x}^{4}}{4 !}$.

vii) Localização de $\mathrm{S}_{\mathrm{n}}(\mathrm{x})$ :

A construção acima pode ser feita de modo geral o que permite localizar os elementos $\mathrm{S}_{\mathrm{n}}(\mathrm{x})$, para todo $n \in \mathbb{N}$, no eixo dos cossenos, o que conclui a prova.

\section{Referências}

[1] Figueiredo, D. G. Análise Real, LTC, 1996.

[2] Silva Júnior, Luís Pereira da, Construções Geométricas Por Régua e Compasso e Números Construtiveis. Trabalho de Conclusão de Curso - Universidade Federal de Campina Grande, Centro de Ciências e Tecnologia Profmat, 2013.

[3] Putnoki, José Carlos, Elementos de Geometria e Desenho Geométrico, Vol. 1, Ed. Scipione, 1989.

[4] Putnoki, José Carlos, Elementos de Geometria e Desenho Geométrico, Vol. 2, Ed. Scipione, 1989.

[5] Lima, E. L., Análise Real, Vol 1, Coleção Matemática Universitária, Impa, 8ª ed., 2006.

[6] Muniz Neto, A. C., Fundamentos de Cálculo, Coleção Profmat, SBM, $1^{\text {a }}$ ed., 2015. 
Daniela Maria Grande Vicente Universidade Estadual do Oeste do Paraná <daniela.grande@unioeste.br>

André Vicente Universidade Estadual do Oeste do Paraná <andre.vicente@unioeste.br>

Recebido: $30 / 11 / 2020$

Publicado: $02 / 03 / 2021$ 